\title{
Down-Regulation of miR-327 Alleviates Ischemia/Reperfusion-Induced Myocardial Damage by Targeting RP105
}

\author{
Ying Yang ${ }^{b}$ Jun Yang ${ }^{a} \quad$ Xiao-wen Liu ${ }^{b} \quad$ Jia-wang Ding ${ }^{a}$ Song Li $^{\mathrm{a}}$ \\ Xin Guoc Chao-jun Yang ${ }^{d}$ Zhi-xin Fan ${ }^{a}$ Hui-bo Wang ${ }^{c}$ Qi Lib \\ Hui-min Wang ${ }^{\mathrm{e}}$ Jian Yang ${ }^{\mathrm{a}}$
}

aDepartment of Cardiology, The First College of Clinical Medical Sciences, China Three Gorges University, Yichang, 'bnstitute of Cardiovascular Diseases, China Three Gorges University, Yichang, 'Department of Cardiology, Renmin Hospital of Wuhan University, Wuhan, dDepartment of Emergency and Critical Care Medicine, Yichang Central People's Hospital, Yichang, eInstitute of biology and pharmacy, China Three Gorges University, Yichang, China

\section{Key Words}

miR-327 • Myocardial ischemia/reperfusion injury •Inflammation • RP105 • TLR4 • TLR2

\begin{abstract}
Background/Aims: Micro RNAs (miRNAs) play a very important role in myocardial ischemia/ reperfusion injury (MIRI), including in inflammation, apoptosis, and angiogenesis. Previous studies have demonstrated up-regulation of miR-327 in renal ischemia/reperfusion injury and MIRI. Via TargetScan, we found RP105 is a possible target gene of miR-327; our previous studies have also confirmed that RP105 acted as a cardioprotective protein in MIRI by reducing inflammation. However, the regulatory effect of miR-327 on RP105 has not previously been proposed. In our study, we aimed to identify the regulatory effect of miR-327 on RP105 protein in MIRI rats. Methods: Sixty male Sprague-Dawley rats were randomly divided into five groups, which were pre-treated with saline (sham and ischemia/reperfusion group), adenovirus-expressing miR-327-RNAi (Ad-miR-327-i group), control (Ad-NC group), or primiR-327 (Ad-miR-327 group) treatments. Three days later, the rat MIRI model was established by ischemia for $30 \mathrm{~min}$, followed by reperfusion for $3 \mathrm{~h}$. Myocardium and plasma were harvested and assessed. Results: miR-327 was increased by nearly 3-fold both in myocardium and plasma, which down-regulated RP105 in a 3'-untranslated region-dependent manner, and down-regulation of miR-327 via adenovirus transfection indirectly suppressed the TLR4/ TLR2-MyD88-NF-KB signaling axis activation via up-regulation of RP105, which subsequently resulted in reduced myocardial infarct size, attenuated cardiomyocyte destruction, and alleviated inflammation. In contrast, up-regulation of miR-327 induced the opposite effect.
\end{abstract}

Y. Yan and J. Yang contributed equally to this work.

\begin{tabular}{ll}
\hline Jun Yang & Department of Cardiology, The First College of Clinical Medical Sciences \\
and Jian Yang & China Three Gorges University, No. 183, Yiling Road, Yichang City, Hubei Province (PR China) \\
& Tel. +8615549309210, Fax $+86(0717) 6482302$, E-Mail yangjun@ctgu.edu.cn; yangjian@ctgu.edu.cn
\end{tabular}


Conclusion: Down-regulation of miR-327 exerts a cardioprotective effect against MIRI by reducing inflammation, which may constitute a promising molecular therapeutic target for treating MIRI.

(C) 2018 The Author(s)

Published by S. Karger AG, Basel

\section{Introduction}

Myocardial infarction, a coronary acute occlusion that results in a sudden lack of blood and oxygen supply to cardiomyocytes, is a major cause of morbidity and mortality in both developed and developing countries. Timely and effective reperfusion therapy, such as percutaneous coronary intervention, thrombolysis, and coronary artery bypass grafting, contributes to declines in disability and mortality. However, restoration of the blood flow can induce even worse microstructural destruction, termed myocardial ischemia/reperfusion injury (MIRI), which lessens the beneficial effect of reperfusion therapy [1]. Recently, a series of in vitro and in vivo studies have demonstrated that innate inflammation, apoptosis, autophagy, platelet activation, and oxidative stress contribute to MIRI [2-6]. However, there is no effective strategy for limiting or preventing MIRI, so a new molecular therapeutic target is necessary.

Innate inflammation plays a key role in MIRI. Recent studies show that ischemic cardiomyocytes can release pathogen-associated molecular patterns (PAMPs), which interact with pattern recognition receptors (PRRs) [7]. Among these PRRs, Toll-like receptors (TLRs) have been identified as important elements. So far, at least 10 TLRs have been identified in mammalian species and 5 of these (i.e., TLR1, TLR2, TLR4, TLR5, and TLR6) are located on the cell surface and are activated by diverse PAMPs. Among these TLRs, TLR4 and TLR2 are mostly expressed in myocardium $[7,8]$. TLR4 and TLR2 are type I transmembrane proteins that have a leucine-rich repeat extracellular domain and an intracellular Toll-interleukin (IL)1 receptor (TIR) domain, which interact with universal adaptor proteins termed myeloid differentiation factor 88 (MyD88), and a TIR domain containing adaptor inducing interferon (IFN)- $\beta$ (TRIF). Once activated, the two main pathways, namely, the Myd88-dependent and TRIF-dependent signaling pathways, are initiated, leading to nuclear factor (NF)- $\mathrm{BB}$ and interferon regulatory factor- 3 activation and eventually the production of pro-inflammatory factors, like IL-6, tumor necrosis factor (TNF)- $\alpha$, and IFN- $\beta$ [7, 9]. Radioprotective $105 \mathrm{kDa}$ protein (RP105), termed CD180 in immunology and also a member of the TLRs family, possesses only 6-11 intracellular amino acids and lacks a TIR domain [10]. Studies have shown that two small glycoproteins called MD-2 and MD-1 are involved in TLR4 and RP105 activation, respectively, to form heterodimers (TLR4/MD-2 and RP105/MD-1), while TLR2 forms heterodimers with TLR1 or TLR6 [8, 10-12]. RP105 (-/-) B cells respond poorly to not only the TLR4 ligand lipopolysaccharide (LPS) but also TLR2 ligand lipoproteins, and RP105 knock-out mice were severely impaired in the production of antibodies against LPS or lipoproteins, indicating that RP105 is involved in inflammation in cooperation with TLR4 and TLR2 [12]. In macrophages and dendritic cells, RP105 has been recognized as a specific inhibitor of the TLR4 pathway, while the effect of RP105 in the TLR2 pathway remains controversial $[13,14]$. In a rat model, RP105 is markedly reduced in ischemia/reperfusion (I/R) myocardium, and up-regulation of RP105 decreases infarct size and attenuates myocardial inflammation, apoptosis, and autophagy via the inhibition of the TLR4 pathway $[3,4]$. Our unpublished data show that up-regulation of RP105 also results in suppression of the TLR2 pathway, in accordance with the TLR4 pathway. These findings indicate that RP105 attenuates MIRI by inhibiting the TLR4 and TLR2 pathways.

MicroRNAs (miRNAs) are approximately 20 to 22 nucleotides long and are capable of degrading target mRNAs by base pairing with the $3^{\prime}$ - untranslated regions (3'-UTRs). Recent research has demonstrated that miRNAs play an essential role in MIRI [15-20]. For example, miR-199a-5p, miR-15, and miR-92a aggravate MIRI, while miR-17-3p, miR-210, and miR499 are cardioprotective miRNAs [15-20]. Interestingly, miR-327 is also obviously increased in the myocardial microvascular endothelial cells of diabetic rat models as well as within 
I/R heart tissues and renal I/R injury models [21-23]. A search using the public prediction algorithm TargetScan (http://www.targetscan.org/) shows that RP105 is a possible target gene of miR-327. Furthermore, we performed luciferase assays in 293T cells, confirming the down-regulation of RP105 by miR-327 in a 3'-UTR-dependent manner. Based on these results, we postulated that down-regulation of miR-327 could indirectly suppress the TLR4 and TLR2 signaling pathways by targeting RP105 to attenuate MIRI.

\section{Materials and Methods}

Animals

Sixty adult male Sprague-Dawley rats weighing 220-250 g (specific-pathogen-free grade) were purchased from the Animal Experiment Center of China Three Gorges University. All experimental procedures were conducted in conformity with National Institutes of Health (NIH) guidelines (NIH Pub. No. 8523, revised 1996) and were approved by the Animal Care and Use Committee of China Three Gorges University. All efforts were made to minimize animal suffering.

\section{MiR-327 target prediction and luciferase assays}

The potential targets of miR-327 were identified using TargetScan. Luciferase assays were performed using $293 \mathrm{~T}$ cells with the firefly luciferase report vector $(0.1 \mu \mathrm{g})$, miRNA expression plasmid $(0.4 \mu \mathrm{g})$, and the control vector containing Renilla luciferase $(0.02 \mu \mathrm{g})$ in a 24-well plate, according to the protocol of the luciferase assay kit used (E2910, Promega, Fitchburg, WI). Forty-eight hours later, the luciferase activity was assayed with the Dual-Luciferase ${ }^{\circledR}$ Reporter Assay System (Promega) and was normalized to Renilla luciferase activity.

\section{Experimental design}

Rats were adaptively fed under suitable relative humidity, temperature, and light cycle conditions with free access to water and food for 1 week [6]. Then, the rats were randomly divided into five groups $(n=$ 12), namely, the sham-operation with normal saline (NS) group (sham group), the myocardial I/R with NS group (I/R group), the myocardial I/R with Ad-miR-327-RNAi group (Ad-miR-327-i group), the myocardial I/R with Ad-NC group (Ad-NC group), and the myocardial I/R with Ad-miR-327 group (Ad-miR-327 group; Fig. 1).

Adenoviral vector construction
and transfection
The adenoviral vectors were

The adenoviral vectors were begins with the miR-327 RNAi or pri-miR-327 oligonucleotides being designed and cloned into the AdMax adenovirus system (Microbix Biosystems, Mississauga, Canada) with the vectors GV201 or GV202 and hU6-MCS-CMV-EGFP. Recombinant adenoviruses were picked and propagated in HEK293 cells (American Type Culture Collection, Manassas, VA). The virus was then purified using an Adeno-XTM Virus Purification Kit (BD Biosciences; Clontech, Mountain View, CA), titrated to achieve $2 \mathrm{E}+10$ $\mathrm{PFU} / \mathrm{mL}$, and stored at $-70^{\circ} \mathrm{C}$.

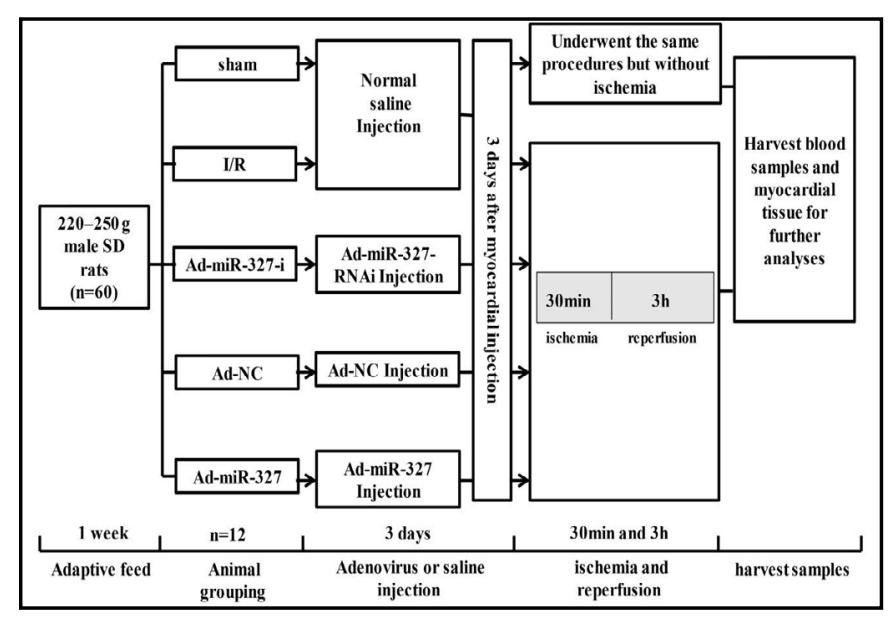

Fig. 1. Experiment schematic diagram. The establishment of the MIRI model included five major steps: adaptive feeding (1 week), animal grouping (five groups: sham group, I/R group, Ad-miR327-i group, Ad-NC group, and Ad-miR-327 group), adenovirus or saline injection (3 days), ischemia (30 min) and reperfusion ( $3 \mathrm{~h})$, and lastly, harvesting samples. 


\section{Cellular Physiology Cell Physiol Biochem 2018;49:1090-1104

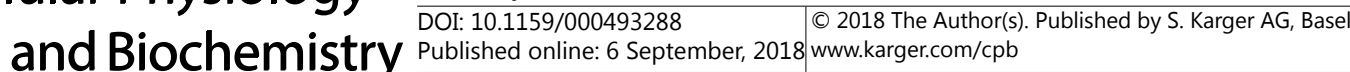

Yang et al.: Down-Regulation of Mir-327 Attenuates MIRI

Rats were anesthetized with sodium pentobarbital $(40 \mathrm{mg} / \mathrm{kg}$ ) by intra peritoneal injection and ventilated with a small animal ventilator (Model ZH-DW-3000A/B, Zhenhua Teaching Instrument Co., Ltd, Yuanyang, China; tidal volume, $20 \mathrm{~mL} / \mathrm{kg}$; respiratory rate, 85 times/min; inspiration/expiration, 2/1). The chests of the rats were opened through the fourth and fifth ribs to expose the heart and cut open the pericardium. Then, $150 \mu \mathrm{L}\left(1.0 \times 10^{10} \mathrm{PFU} / \mathrm{mL}\right)$ of Ad-miR-327-RNAi, Ad-miR-327, Ad-NC, or NS were injected into the cardiac apex (at 4-6 spots) with a 30-gauge needle, and the chest was closed rapidly.

\section{Assessment of adenovirus transfection efficiency}

We assessed the efficiency of adenovirus transfection by immunofluorescence microscopy. Three days after the cardiac apex injection, but without ischemia, myocardial tissue was collected, fixed in $4.0 \%$ paraformaldehyde, embedded in paraffin, and then sectioned. Sections were dewaxed and washed three times in phosphate-buffered saline, and the cell nuclei were stained with 4', 6'-diamidino-2-phenylindole (DAPI). Samples were then mounted with medium containing anti-fluorescence quenching agent. Finally, images were captured with a fluorescence microscope (BX51, Olympus America, Center Valley, PA) at 200x and $400 \times$ magnification.

\section{Establishment of rat MIRI model}

Three days later, rats were re-anesthetized and re-ventilated with a small animal ventilator, using procedures that have been described previously [6]. Briefly, the chest was reopened, and the origin of left anterior descending (LAD) artery was identified. Then a 6-0 silk suture was made around the LAD, and medical latex tubing (diameter, $2 \mathrm{~mm}$; length, $5 \mathrm{~mm}$ ) was placed between the LAD artery and the silk suture. MIRI was induced by ischemia for $30 \mathrm{~min}$ (tightening the suture), followed by reperfusion for $3 \mathrm{~h}$ (clipping the suture). The sham group underwent the same procedures, except for tightening of the ligature. MIRI models were defined as successful by observing a blanched appearance of the ischemic region, and an elevation of an electrocardiogram S-T segment. When reperfusion was finished, blood samples were obtained and rats were sacrificed so that the cardiac apex tissue could be harvested for further analyses.

\section{Detection of myocardial enzymes}

After $3 \mathrm{~h}$ of reperfusion, blood samples from the inferior vena cava, were immediately obtained without an anticoagulant tube, and then serum was separated by centrifugation at $3000 \mathrm{rpm}$ for $10 \mathrm{~min}$ at $4^{\circ} \mathrm{C}$. Creatine kinase-muscle/brain (CK-MB) and lactate dehydrogenase (LDH) were tested using an ADVIA2400 automatic biochemical analyzer (Siemens, Berlin, Germany) at Yichang Central People's Hospital.

\section{Assessment of myocardial infarct size}

The myocardial infarct size was evaluated by 2, 3,5-triphenyltetrazolium chloride (TTC; Sigma-Aldrich, St. Louis, MO) staining. The procedure has been described previously [24]. Briefly, after $3 \mathrm{~h}$ of reperfusion, the inferior vena cava was isolated. Then, $1 \mathrm{~mL}$ of $1.5 \%$ TTC phosphate-buffered saline was injected into inferior vena cava and then incubated for $15 \mathrm{~min}$. Hearts were removed and washed with cold saline and stored at $-80^{\circ} \mathrm{C}$ for $5 \mathrm{~min}$. Samples were then sliced into five sections from the apex to the base and fixed in $4 \%$ paraformaldehyde overnight. The infarct area (white) and the normal area (red) could be distinguished and were measured using Image-Pro Plus 6.0 software (Media Cybernetic, Rockville, MD).

\section{Histopathology}

After $3 \mathrm{~h}$ of reperfusion, myocardial tissue was collected and fixed in $4.0 \%$ paraformaldehyde, embedded in paraffin, and sectioned into 4- $\mu \mathrm{m}$ slices. Samples were then stained with hematoxylin and eosin (H\&E; hematoxylin: No. H9627, Sigma-Aldrich; eosin: No. 71014544, Sinopharm Group Co., Ltd, Shanghai, China) and examined under a light microscope (Olympus BX53, Olympus America) at 200× magnification.

\section{Quantitative real-time PCR}

Total RNA was isolated from myocardial tissue and plasma with Trizol Reagent (Aidlab Biotechnologies., Ltd, Beijing, China) according to the manufacturer's instructions. The ratio of OD260 and OD280 was used to determine the concentration and purity of total RNA samples. Then, the RNA was reversed transcribed into cDNA using a reverse transcription kit (Takara, Beijing, China) following the manufacturer's instructions. Then, quantitative real-time PCR (qRT-PCR) was performed with a SYBR green/fluorescein qPCR Master Mix 
kit (2×) (Vazyme Biotech Co., Ltd, Nanjing, China) with the ABI Prism 7500 system (PE Applied Biosystems, Foster City, CA). The conditions were as follows: $50^{\circ} \mathrm{C}$ for 2 $\min$; $95^{\circ} \mathrm{C}$ for $10 \mathrm{~min}$; and 40 cycles of $95^{\circ} \mathrm{C}$ for $30 \mathrm{~s}$ and $60^{\circ} \mathrm{C}$ for $30 \mathrm{~s}$. U6 and GAPDH mRNA levels were determined as an internal control; miR-327 levels were normalized to U6, while RP105, TLR2, TLR4, MYD88, and NF- $\kappa \mathrm{B}$ mRNA expression was normalized to GAPDH. The resulting data were analyzed using the comparative Ct method $\left(2^{-\Delta \Delta C t}\right)$. The primers used to amplify gene products were as follows (Table 1).
Table 1. Primers of miR-327, RP105, TLR2, TLR4, MyD88, and NF- $\kappa$ B were used to amplify gene products, and U6 and GAPDH were determined as internal controls

\begin{tabular}{|c|c|c|c|}
\hline Name & Primer & Sequence & Size \\
\hline \multirow{2}{*}{ U6 } & Forward & 5'- CGCTTCGGCAGCACATATAC - $3^{\prime}$ & \\
\hline & Reverse & 5'- AAATATGGAACGCTTCACGA - $3^{\prime}$ & \\
\hline \multirow{2}{*}{ miR-327 } & Loop-Primer & 5'-GTCGTATCCAGTGCAGGGTCCGAGGTATTCGCACTGGATACGACACCCTCAT-3' & \\
\hline & F-Primer & 5'- TGCGCCCTTGAGGGGCATG -3' & \\
\hline \multirow{2}{*}{ GAPDH } & Forward & 5'- ACAGCAACAGGGTGGTGGAC - $3^{\prime}$ & \multirow{2}{*}{ 253bp } \\
\hline & Reverse & 5'- TTTGAGGGTGCAGCGAACTT -3' & \\
\hline \multirow{2}{*}{ PR105 } & Forward & 5'- GAGCCTGAACCTGAGCTACA - $3^{\prime}$ & \multirow{2}{*}{ 173bp } \\
\hline & Reverse & 5'- GGAGGCAGTGGGAGAGATTT -3' & \\
\hline \multirow{2}{*}{ TLR4 } & Forward & 5'- TATCGGTGGTCAGTGTGCTT -3' & \multirow{2}{*}{$167 \mathrm{bp}$} \\
\hline & Reverse & 5'- CTCGTTTCTCACCCAGTCCT - $3^{\prime}$ & \\
\hline \multirow{2}{*}{ TLR2 } & Forward & 5'- AGGCCCCTTTCCTCTTTGAA - $3^{\prime}$ & \multirow{2}{*}{$214 \mathrm{bp}$} \\
\hline & Reverse & 5'- GGGACTCATAGTTGCCGAGA -3' & \\
\hline \multirow{2}{*}{ MyD88 } & Forward & 5'- CGACGCCTTCATCTGCTACT -3' & \multirow{2}{*}{$172 \mathrm{bp}$} \\
\hline & Reverse & 5'- ATGCGACGACACCTTTTCTC -3' & \\
\hline \multirow{2}{*}{ NF- $\mathrm{kB}$} & Forward & $5^{\prime}-$ TCCAACACAGGCATCACCCA - $3^{\prime}$ & \multirow{2}{*}{$286 \mathrm{bp}$} \\
\hline & Reverse & 5'- CCGTAACCGCGTAGTCGAAG - $3^{\prime}$ & \\
\hline
\end{tabular}

\section{Western blots}

The proteins levels of RP105, TLR4, TLR2, MyD88, and NF- $\kappa B$ in myocardial tissue were measured using western blots [6]. Briefly, myocardial tissue was lysed in RIPA buffer (Beyotime Biotechnology, Jiangsu, China). The protein concentration was measured by a bicinchoninic acid protein assay (Beyotime Biotechnology) and then denatured by boiling. A total of $40 \mu \mathrm{g}$ of protein was separated by electrophoresis in a sodium dodecyl sulfate-polyacrylamide gel and then transferred onto polyvinylidene fluoride membranes (Millipore, Billerica, MA). After being blocked with 5\% non-fat dried milk for $2 \mathrm{~h}$ at room temperature, the membranes were incubated overnight at $4{ }^{\circ} \mathrm{C}$ with each primary antibody (anti-RP105 [1:800, Boster Biological Technology Co., Ltd., Wuhan, China], anti-TLR2 [1:1000, EPIT, Shenzhen, China], anti-TLR4 [1:1000, Proteintech Group, Wuhan, China], anti-MyD88 [1:500, Proteintech Group], anti-NF-kB [1:1000, Affinity, Cincinnati, USA] or anti-GAPDH [1:1000, Goodhere Biotechnology Co., Ltd., Hangzhou, China]). After being washed five times, the blots were then incubated with peroxidase-conjugated secondary antibodies for $2 \mathrm{~h}$ at room temperature. Bands were scanned and analyzed by an electrochemiluminescence detection kit (Thermo Fisher Scientific, Waltham, MA), with GAPDH serving as a loading control.

\section{Enzyme-linked immunosorbent assay}

The levels of IL- 6 and TNF- $\alpha$ in myocardial tissue were measured by an enzyme-linked immunosorbent assay (ELISA) using an IL-6 rat ELISA kit and TNF- $\alpha$ rat ELISA kit (Elabscience Biotechnology Co., Ltd., Wuhan, China) according to the manufacturer's instructions. Briefly, $100 \mu \mathrm{L}$ samples were added to the wells and incubated at $37^{\circ} \mathrm{C}$ for $90 \mathrm{~min}$. Liquid was then removed, and the plate was dried. Later, the Detection antibody solution (Elabscience Biotechnology Co., Ltd., Wuhan, China) was added and incubated at $37^{\circ} \mathrm{C}$ for $1 \mathrm{~h}$. A horseradish peroxidase conjugate solution was added to the washed plate and incubated at $37^{\circ} \mathrm{C}$ for $30 \mathrm{~min}$. Lastly, substrate and termination solutions were added to the wells to stop the reaction, and the color of the solution changed from blue to yellow immediately. The optical density of each well was immediately measured using an automatic microplate reader (Thermo Fisher Scientific) at $450 \mathrm{~nm}$. Amounts of TNF- $\alpha$ and IL-6 were related to $\mathrm{OD}_{450}$.

\section{Statistical analyses}

All statistical analyses were conducted with SPSS 19.0 software (IBM Corp., Armonk, NY). Quantitative data were expressed as the mean \pm standard deviation (SD). Statistical comparisons between two groups were performed using Student's $t$-tests, while those between three or more groups were performed using one-way analysis of variance, followed by the Student-Newman-Keuls $Q$ test. For all statistical analyses, $P<$ 0.05 was considered the threshold of statistical significance. 


\section{Cellular Physiology Cell Physiol Biochem 2018;49:1090-1104 and Biochemistry $\begin{aligned} & \text { DOI: 10.1159/000493288 } \\ & \text { Published online: 6 September, } 2018 \text { www.karger.com/cpb }\end{aligned}$}

Yang et al.: Down-Regulation of Mir-327 Attenuates MIRI

\section{Results}

\section{MiR-327 and RP105 changed inversely in MIRI rat models}

The expression levels of miR-327 in myocardium and plasma were measured by qRTPCR, as shown in Fig. 2A. The level of RP105 in myocardial tissue was measured by qRTPCR and western blot (Fig. 2B). After $3 \mathrm{~h}$ of reperfusion, the expression of miR-327 was significantly higher in the I/R group; conversely, the mRNA and protein levels of RP105 were significantly decreased in I/R myocardial tissue compared with the sham group. These data indicate that miR-327 and RP105 might interact with each other in MIRI rat models.

\section{Adenovirus was successfully transfected into myocardium}

To examine the effect of miR-327 on MIRI, we injected recombinant adenoviruses expressing either miR-327-RNAi or pri-miR-327 into the myocardium. Three days after transfection, the efficiency of adenovirus transfection into the myocardium was determined by qRT-PCR and fluorescent microscopy, as shown in Fig. 2C-E. The decreased and increased miR-327 expression levels were then validated (Fig. 2C-D) and transfection of Ad-miR327-RNAi reduced the expression of miR-327 in myocardium; conversely, Ad-miR-327 upregulated the expression of miR-327, while NS or Ad-NC had no effect on the expression of miR-327 in myocardial tissue, in accordance with the levels in plasma. Fluorescent microscopy showed high green fluorescence in myocardial tissue injected with recombinant adenoviruses, while little background color was observed in myocardium injected with NS (Fig. 2E-F). These results indicate that adenovirus was successfully transfected into myocardial tissue.

Recombinant adenovirus over-expression of miR-327 RNAi reduced MIRI in vivo

To evaluate the effect of miR-327 on MIRI, we infected myocardium with adenovirus vector containing miR-327-RNAi to depress miR-327and pre-miR-327 to overexpress miR327. Three days later, adenovirus was successfully transfected, and then the I/R procedure was conducted. Afterwards, myocardial infarct size, myocardial histopathological changes, serum myocardial enzymes (CK-MB and LDH), and inflammatory factors (IL- 6 and TNF- $\alpha$ ) were examined.

Myocardial infarct size was evaluated by TTC staining (Fig. 3A) and calculated as (white area) / (white area + red area). Approximately $20 \%$ of examined tissues showed myocardial infarct areas in the I/R group, while no myocardial infarcts were observed in the sham group. Moreover, the percentage of infarct areas was reduced to $11 \%$ in the I/R myocardium transfected with Ad-miR-327-RNAi (Ad- miR-327-i vs. I/R group, $P<0.05$ ), and myocardial infarct areas were up to 38\% in I/R myocardium transfected with Ad-miR-327(Ad- miR-327 vs. I/R group, $P<0.05$ ). However, treatment with Ad-NC or NS had no effect on infarct size (Ad-NC vs. I/R group, $P>0.05$ ).

Histopathological changes were observed by H\&E staining (observed at $200 \times$ magnification). As shown in Fig. 3A, the structure and morphology of myocardial tissues were completely maintained in the sham group; in the I/R group, myocardial fibers were damaged with neutrophil infiltration and erythrocytes, enlarged intercellular spaces, extensive edema, and myocyte necrosis. Pathological damage was markedly attenuated in I/R myocardium transfected with Ad-miR-327-RNAi, and damage was aggravated in I/R myocardial tissue transfected with Ad-miR-327. However, treatment with Ad-NC or NS had no effect on histopathological differences.

The levels of CK-MB (Fig. 3C) and LDH (Fig. 3D) in serum were initially low in the sham group, and the I/R group had higher levels of CK-MB and LDH (I/R group vs. sham group, $P$ $<0.05$ ). Moreover, the elevation of serum CK-MB and LDH was significantly suppressed by injection of Ad-miR-327-RNAi (Ad-miR-327-i vs. I/R group, $P<0.05$ ) and resulted in higher levels of CK-MB and LDH by injection of Ad-miR-327 (Ad- miR-327 vs. I/R group, $P<0.05$ ). However, treatment with Ad-NC or NS had no effect on I/R-induced elevation of CK-MB and LDH in serum (Ad-NC vs. I/R group, $P>0.05$ ). 


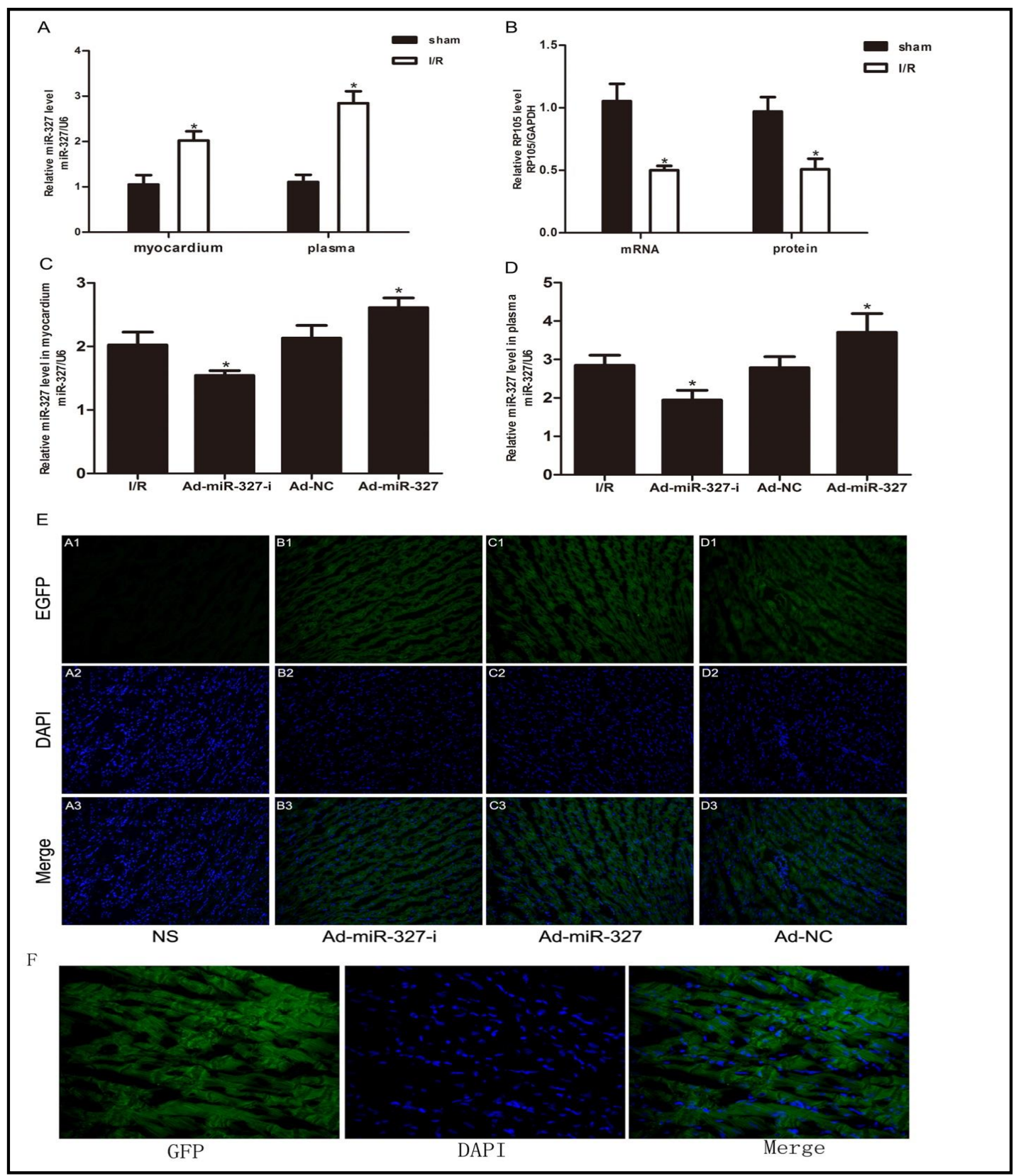

Fig. 2. MiR-327 and RP105 changed inversely in MIRI rat models. (A) qRT-PCR of miR-327 in myocardium and plasma. MiR-327 expression was significantly increased in MIRI rat models. Values are expressed as the mean \pm SD $\left(n=3,{ }^{*} \mathrm{P}<0.05\right)$. (B) mRNA and protein levels of RP105 in myocardial tissue. RP105 levels were significantly decreased in I/R myocardial tissue. Values are expressed as the mean $\pm \mathrm{SD}(\mathrm{n}=3, * \mathrm{P}<0.05)$. (C-D) The level of miR-327 in myocardium and plasma injected with adenovirus or NS. Both in myocardial tissue and plasma, transfection of Ad-miR-327-RNAi reduced the miR-327; conversely, transfection of AdmiR-327 up-regulated the expression of miR-327, while NS or Ad-NC had no effect on miR-327. Values are expressed as the mean $\pm \mathrm{SD}\left(\mathrm{n}=3,{ }^{*} \mathrm{P}<0.05\right.$ vs. I/R or Ad-NC group). (E) Representative photomicrographs of immunofluorescence microscopy after adenovirus or NS injection (200× magnification). EGFP (green, B1-D1), DAPI-labeled nuclei of cardiomyocytes (blue, B2-D2), and merged (B3-D3) images are shown for comparison with an image with only a little background color in myocardial tissue injected with NS (A1). (F) Representative photomicrographs of immunofluorescence microscopy after adenovirus (Ad-NC) injection (400× magnification). EGFP (green), DAPI-labeled nuclei of cardiomyocytes (blue), and merged images are shown. 


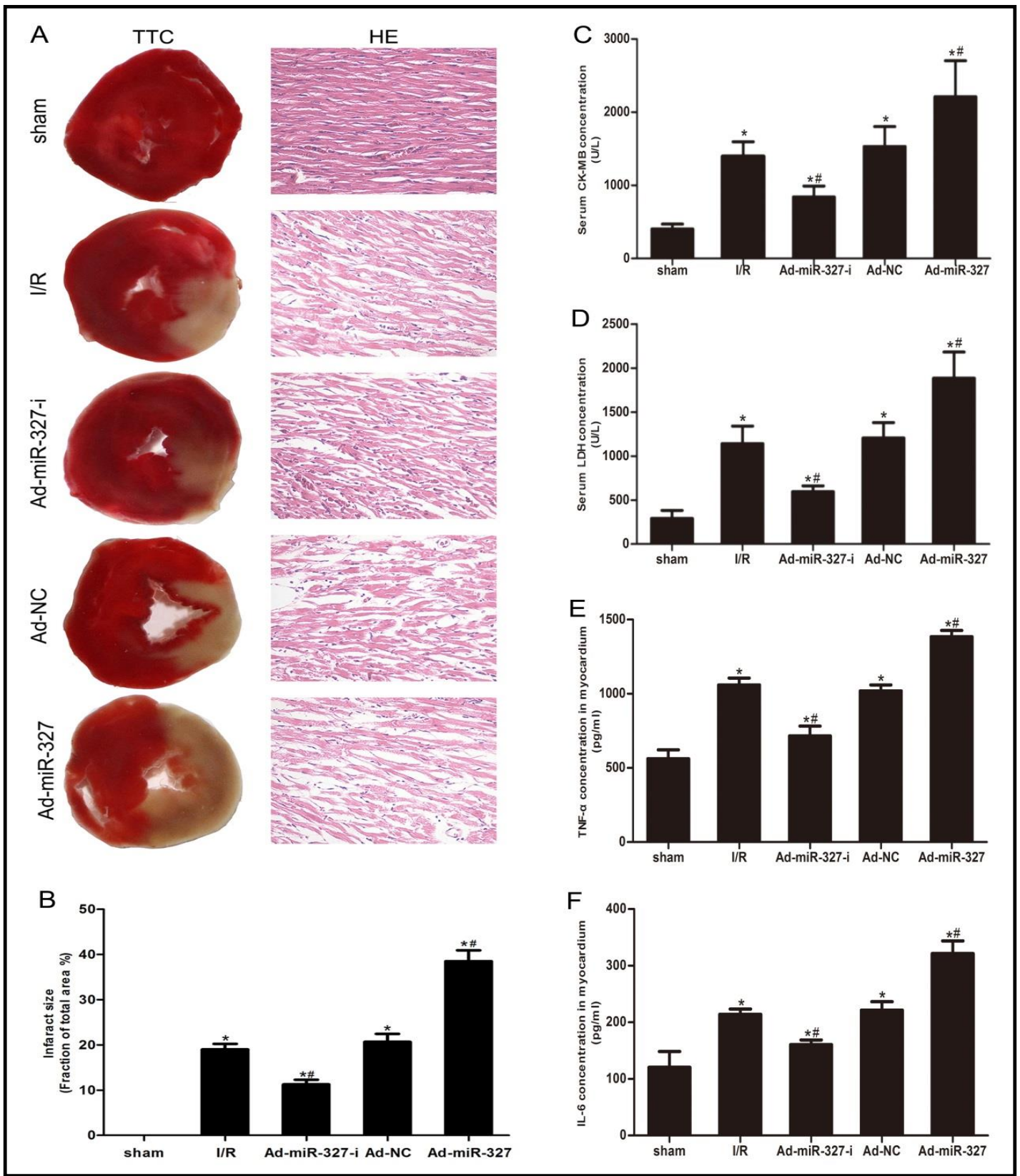

Fig. 3. Down-regulation of miR-327 alleviated MIRI-induced pathological damage and elevations of myocardial enzyme and inflammatory factors. (A) Representative images of TTC staining and H\&E staining (200x magnification). In TTC staining, the red area is non-infarcted tissue, while white area is infarcted tissue. (B) The infarct size is calculated as (white area) / (white area + red area). Values are expressed as the mean $\pm \mathrm{SD}$, ( $\mathrm{n}=5,{ }^{*} \mathrm{P}<0.05$ vs. sham group, ${ }^{\#} \mathrm{P}<0.05$ vs. Ad-NC or I/R group). (C-D) CK-MB and LDH levels were significantly suppressed by Ad-miR-327-RNAi and up-regulated by Ad-miR-327. Values are expressed as the mean $\pm \mathrm{SD},\left(\mathrm{n}=9\right.$, ${ }^{*} \mathrm{P}<0.05$ vs. sham group, ${ }^{*} \mathrm{P}<0.05$ vs. Ad-NC or I/R group). (E-F) TNF- $\alpha$ and IL-6 levels were significantly suppressed by Ad-miR-327-RNAi and up-regulated by Ad-miR-327. Values are expressed as the mean $\pm \mathrm{SD},\left(\mathrm{n}=3,{ }^{*} \mathrm{P}<0.05\right.$ vs. sham group, ${ }^{\#} \mathrm{P}<0.05$ vs. Ad-NC or I/R group). 
Inflammatory factors TNF- $\alpha$ (Fig. 3E) and IL-6 (Fig. 3F) were significantly up-regulated in myocardium that had been subjected to I/R injury (I/R vs. sham group, $P<0.05$ ). Moreover, the elevation of TNF- $\alpha$ and IL- 6 was significantly suppressed by injection of Ad-miR-327RNAi (Ad-miR-327-i vs. I/R group, $P<0.05$ ) and increased by injection of Ad-miR-327(AdmiR-327 vs. I/R group, $P<0.05$ ). However, treatment with Ad-NC or NS had no effect on I/R-induced elevation of IL- 6 and TNF- $\alpha$ (Ad-NC vs. I/R group, $P>0.05$ ).

\section{MiR-327 down-regulated RP105 in a 3'-UTR-dependent manner}

Via a bioinformatic search, we found RP105 was a potential target of miR-327 in a 3 '-UTR-dependent manner. To test this, we performed luciferase assays, as shown in Fig. 4A. Compared with the group without the RP105 3'-UTR, the luciferase activity of the WT RP105 3'-UTR was significantly reduced by miR-327, indicating that miR-327 may target RP105 protein in a 3'-UTR-dependent manner. Furthermore, the mRNA level of RP105 was notably increased in I/R myocardium transfected with Ad-miR-327-RNAi (Ad-miR-327-i vs. I/R group, $P<0.05$ ), while the levels were significantly decreased in I/R myocardial tissue transfected with Ad-miR-327 (Ad-miR-327 vs. I/R group, $P<0.05$ ). To further confirm these results, we measured RP105 protein concentrations, which were similar to its mRNA levels. The titers of RP105 were decreased and increased, respectively, by Ad-miR-327 and Ad-miR327-RNAi, and treatment with Ad-NC or NS had no effect on the levels of RP105 (Ad-NC vs. I/R group, $P>0.05$; Fig. 4B,C). These data indicate that miR-327 targets RP105 in a $3^{\prime}$-UTRdependent manner.

Down-regulation of miR-327 attenuated MIRI via suppression of the TLR4/TLR2-MyD88$N F-\kappa B$ pathway

To validate the hypothesis that the TLR4/TLR2-MyD88-NF- $\kappa B$ pathway was suppressed to attenuate MIRI when miR-327 is down-regulated, we next examined the mRNA and protein concentrations of TLR4, TLR2, MyD88, and NF-KB. As shown in Fig. 5, levels of TLR4, TLR2, MyD88, and NF- $\kappa B$ were significantly increased in the I/R group (I/R group vs. sham group,

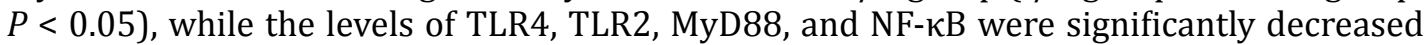
in the I/R myocardium transfected with Ad-miR-327-RNAi (Ad-miR-327-i vs. I/R group, $P$ $<0.05$ ); conversely, these expression levels were notably increased in the I/R myocardium transfected with Ad-miR-327 (Ad-miR-327 vs. I/R group, $P<0.05$ ). However, transduction with Ad-NC or NS had no significant effect on the action of the TLR4/TLR2-MyD88-NF- $\mathrm{BB}$ pathway (Ad-NC vs. I/R group, $P>0.05$ ), indicating that down-regulation of miR-327 could attenuate MIRI via suppression of the TLR4/TLR2-MyD88-NF- KB pathway.

Fig. 4. MiR-327 targets $\mathrm{RP} 105$ in a 3'-UTR-dependent manner. (A) The relative luciferase activity of the wildtype RP105 3'-UTR was significantly reduced by miR-327. Values are expressed as the mean $\pm S D$, $(n=3$, ${ }^{*} \mathrm{P}<0.05$ vs. $3^{\prime}$-UTR-NC group). (B-C) The mRNA and protein levels of RP105 were notably increased by Ad-miR-327RNAi and decreased by Ad-miR-327. Values are expressed as the mean $\pm \mathrm{SD}$, ( $\mathrm{n}=3,{ }^{*} \mathrm{P}<0.05$ vs. sham group, ${ }^{\#} \mathrm{P}<0.05$ vs. Ad-NC or I/R group).

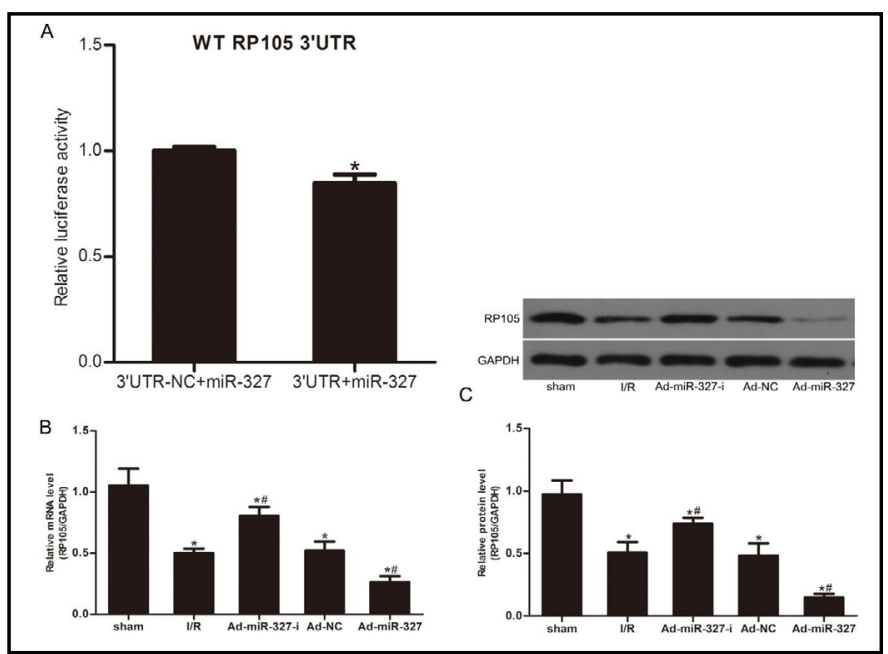




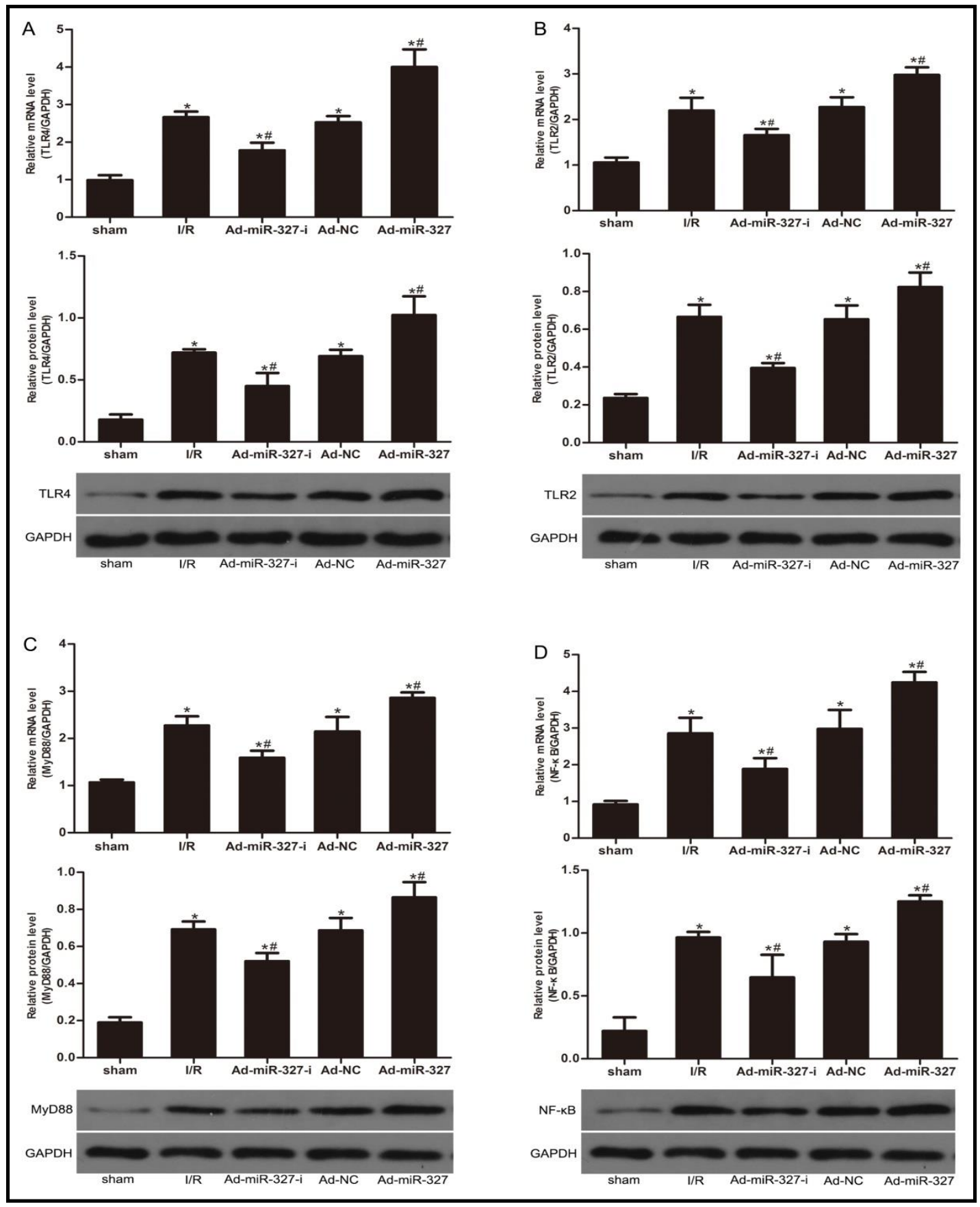

Fig. 5. Down-regulation of miR-327 suppressed the TLR4/TLR2-MyD88-NF- $\mathrm{B}$ pathway in MIRI, while upregulation of miR-327 showed an enhancing effect. (A) The mRNA and protein levels of TLR4. (B) The mRNA and protein levels of TLR2. (C) mRNA and protein levels of MyD88. (D) The mRNA and protein levels of NF$\kappa B$. Values are expressed as the mean $\pm S D,\left(n=3,{ }^{*} \mathrm{P}<0.05\right.$ vs. sham group, ${ }^{*} \mathrm{P}<0.05$ vs. Ad-NC or I/R group).

\section{Discussion}

In this study, we investigated the role of miR-327 in I/R-induced myocardial damage. First, we found that miR-327 was up-regulated and RP105 was down-regulated in I/R myocardial tissue compared with that of the sham group, with an inverse correlation between miR327 and RP105, indicating that miR-327 and RP105 might interact with each other in I/R KARGER 
myocardial tissue. Second, we determined the effects of miR-327 on I/R myocardial tissue by measuring the myocardial infarct size, myocardial histopathological changes, serum myocardial enzymes, and inflammatory factors in I/R myocardial tissue transfected with Ad-miR-327-RNAi, Ad-miR-327, Ad-NC, or NS. The results revealed that down-regulation of miR-327 rapidly reduced myocardial infarct size, attenuated cardiomyocyte destruction, and alleviated inflammation. By contrast, elevation of miR-327 showed the opposite effect. These observations suggested that down-regulation of miR-327 could alleviate MIRI. Finally, via a bioinformatics search and luciferase assays, we identified for the first time that RP105 is a direct target of miR-327. Furthermore, our studies showed that down-regulation of miR327 could indirectly suppress the TLR4/TLR2-MyD88-NF- $\kappa B$ signaling axis. These results suggested that down regulation of miR-327 indirectly suppressed TLR4/TLR2-MyD88-NF- $\kappa B$ signaling axis activation in MIRI rat models via up-regulation of RP105, which subsequently reduced inflammation.

Neutrophils, monocytes, and macrophages are widely recognized as having key roles in innate inflammation. Recent studies suggest that cardiomyocytes may act as immunocytes rather than target cells [7]. When cardiomyocytes were subjected to ischemia, a variety of PAMPs (heat-shock proteins, HMGB1, and reactive oxygen species) released and interacted with PRRs [7, 25]. Among these PRRs, TLRs have been recognized as key elements in myocardial acute inflammation, especially TLR4 and TLR2, which have two common downstream signaling pathways, MyD88-dependent and TRIF-dependent signaling pathways. In MyD88-dependent signaling, once TLR4/TLR2 signaling is stimulated, the TIR domain-containing adaptor protein (TIRAP/Mal) serves as a bridge connecting TLRs and MyD88; then, MyD88 interacts with a series of protein kinases, eventually allowing the released NF- $\kappa \mathrm{B}$ to translocate into the nucleus and promote the production of inflammatory factors. Numerous studies have demonstrated that TLR4 plays a detrimental role in MIRI, while the effect of TLR2 still remains controversial [26-34]. In 2004, Oyama and colleagues reported for the first time that TLR4 served a pro-inflammatory role in rat MIRI models [25]. Subsequently, several investigations also confirmed that TLR4 signaling was significantly up-regulated in ischemic myocardium, and the inhibition of TLR4 signaling could decrease myocardial infarct size, suppress the inflammatory response, and alleviate cardiomyocyte apoptosis [27-30]. However, the role of TLR2 in MIRI still remains uncertain. Some studies have shown that TLR2 activation may accelerate inflammation, fibrosis, and apoptosis [31, 32], while Shishido and colleagues reported that myocardial infarct size and inflammation in infarct area had no difference between wild-type mice and TLR2 deficient mice [33]. Moreover, the MIRI rat model treated with TLR2 ligands (PGN or Pam3CSK4) even induced a cardioprotective effect [34]. In our study, we found TLR2 increased in accordance with TLR4, so we speculated that TLR2 plays a detrimental role in MIRI, similar to that of TLR4.

RP105 is a TLR family, cell-surface protein that lacks a TIR domain. In B cells, RP105 acted as co-stimulator of TLR4 and TLR2 $[12,35]$. However, in macrophages and dendritic cells, RP105 acted as a physiological, endogenous inhibitor of TLR4 $[13,14]$. However, there are no relevant studies that have confirmed the regulation of TLR2 by RP105. Recently, RP105 has been regarded as a cardioprotective TLR in MIRI via suppression of TLR4-related signaling. In our previous research, we found that the expression of RP105 declined dramatically in ischemic myocardial tissue, and over-expression of RP105 via adenovirus transfection reduced myocardial infarct size and attenuated myocardial inflammation, apoptosis, and autophagy by suppression of TLR4 signaling [3, 4, 36]. Additionally, Louwe and colleagues verified that RP105-deficient mice exhibited enhanced inflammation and more pronounced cardiac dilatation after myocardial infarction when compared with wild-type mice [37]. Our unpublished data showed that over-expression of RP105 via adenovirus transfection suppress the TLR2 pathway, in accordance with the TLR4 pathway. In the current study, we used RNA interference technology to indirectly elevate or reduce RP105 expression. Our data demonstrated that over-expression of RP105 could restrain TLR4 and TLR2 signaling at the same time.

\section{KARGER}




\section{Cellular Physiology Cell Physiol Biochem 2018;49:1090-1104 and Biochemistry $\begin{gathered}\text { DOI: 10.1159/000493288 } \\ \text { Published onnine: 6 September, } 2018 \text { www.karger.com/cpb }\end{gathered}$ \\ Yang et al.: Down-Regulation of Mir-327 Attenuates MIRI}

Accumulating evidence indicates that miRNAs play key roles in MIRI, including inflammation, apoptosis, and angiogenesis [15-20]. In 2009, Wang and colleagues reported that MiR-327 expression levels were up-regulated in type-2 diabetic rat models using a miRNA microarray [21]. Subsequently, studies have shown that miR-327 increased by 10-fold in rats with MIRI and nearly 3-fold in the kidneys of rats with renal I/R injury, respectively $[22,23]$. Additionally, in injured sciatic nerves, miR-327 was increased and involved in peripheral nerve regeneration by targeting CCL2 in a 3'-UTR-dependent manner [38]. However, the molecular mechanism for miR-327 regulation on I/R injury remains unclear. In our rat MIRI models, miR-327 was increased by nearly 3-fold both in myocardium and plasma, consistent with previous research $[21-23,38]$. To the best of our knowledge, this is the first report describing the molecular mechanism by which miR-327 regulates MIRI. We performed luciferase assays to confirm that RP105 was a target of miR-327 through directly binding to its $3^{\prime}$-UTR. In the present study, we used adenovirus transfection technology to down-regulate or up-regulate the expression of miR-327 in rat myocardial tissue subjected to I/R injury. Our data show that down-regulation of miR-327 in myocardium increased the expression of RP105, while reducing myocardial infarct size, attenuating cardiomyocyte destruction, and alleviating inflammation. In contrast, up-regulation of miR-327 exhibits the opposite effect. All these data indicated that miR-327 is involved in MIRI regulation via targeting RP105 in a 3'-UTR-dependent manner.

Furthermore, we found an interesting phenomenon, MiR-327 was significantly increased in I/R myocardium and plasma, with an even more obvious increase in plasma. However, it remains unclear where plasma miR-327 is derived from as well as why it is more abundant in plasma. We know exosomes are intriguing signaling intermediates with cargo rich in small RNA molecules, especially miRNAs. The composition of exosomes is not determined randomly, but rather uniquely regulated by cell sources and environmental stressors $[39,40]$. Cardiosphere-derived cells (CDCs) secrete exosomes in a form of highly selective transfer of miRNA into CDC exosomes; that is, the levels of miR-181a, but not miR-181b, were higher in human CDCs, and CDCs exosomes were highly enriched for miR-181b, but not miR-181a [40]. Additionally, in remote ischemic preconditioning, miR-144, which is a cardioprotective miRNA, was transferred via exosomes [41]. Therefore, we speculate that, when I/R injury occurs, miR-327 is selectively transferred into exosomes, which may constitute the main source of plasma miR-327. Accordingly, it remains necessary to analyze the source of plasma miR-327 and determine if it can be used as an early marker for myocardial damage or a prognostic marker for post-MIRI.

However, there are some limitations in our study. First, the effect of TLR2 signaling in MIRI is controversial [31-34]; we mainly used adenovirus transfection technology to regulate the expression of miR-327, but did not suppress TLR4 or TLR2 signaling to exam the effect, respectively. Second, we confirmed the effect of miR-327 on myocardium subjected to I/R injury in vivo but did not verify the effect on hypoxia/reoxygenation cardiomyocytes; we intend to conduct these follow-up experiments in future research. Third, we just detected the effect on the TLR4/TLR2-MyD88-NF- $\mathrm{BB}$ pathway, and the regulation of TRIF-dependent signaling, apoptosis, and autophagy are still unclear. Fourth, we pre-treated rats with adenovirus to regulate gene expression before the I/R injury, which is not suitable for clinical patients, so there is still some work to be done.

\section{Conclusion}

In conclusion, this study demonstrates that the targeted down-regulation of miR-327 may alleviate I/R-induced myocardial damage by targeting RP105 to reduce inflammation in rat models. Thus, miR-327 may constitute a promising molecular therapeutic target for treating MIRI. 


\section{Cellular Physiology Cell Physiol Biochem 2018:49:1090-1104 and Biochemistry \begin{tabular}{l|l} 
DOI: 10.1159/000493288 & (c)18 The Author(s). Published by S. Karger AG, Basel \\
www.karger.com/cpb
\end{tabular}}

Yang et al.: Down-Regulation of Mir-327 Attenuates MIRI

\section{Acknowledgements}

This work was supported by the National Natural Science Foundation of China (Grant Nos. 81670333, 81470387, 81770360, and 81500230) and Hubei Province's Outstanding Medical Academic Leader Program, China.

\section{Disclosure Statement}

The authors declare no conflict of interests.

\section{References}

1 Yellon D M, Hausenloy D J: Myocardial reperfusion injury. N Engl J Med 2007;357:1121-1135.

2 DeBerge M, Yeap XY, Dehn S, Zhang S, Grigoryeva L, Misener S, Procissi D, Zhou X, Lee DC, Muller WA, Luo $\mathrm{X}$, Rothlin C, Tabas I, Thorp EB: MerTK cleavage on resident cardiac macrophages compromises repair after myocardial ischemia reperfusion injury. Circ Res 2017;121:930-940.

3 Li X, Yang J, Yang J, Dong w, Li S, Wu H, Li L: RP105 protects against myocardial ischemia-reperfusion injury via suppressing TLR4 signaling pathways in rat model. Exp Mol Pathol 2016;100:281-286.

4 Guo X, Jiang H, Yang J, Chen J, Yang J, Ding JW, Li S, Wu H, Ding HS: Radioprotective 105 kDa protein attenuates ischemia/reperfusion-induced myocardial apoptosis and autophagy by inhibiting the activation of the TLR4/NF-kappaB signaling pathway in rats. Int J Mol Med 2016;38:885-893.

5 Zhou H, Li D, Zhu P, Hu S, Hu N, Ma S, Zhang Y, Han T, Ren J, Cao F, Chen Y: Melatonin suppresses platelet activation and function against cardiac ischemia/reperfusion injury via PPAR $\gamma /$ FUNDC1/mitophagy pathways. J Pineal Res 2017;63:e12438.

6 Wang H, Yang J, Ding J, Chen L, Li S, Liu X, Yang C, Fan Z, Yang J: RNAi-Mediated Down-Regulation of CD47 Protects against Ischemia/Reperfusion-Induced Myocardial Damage via Activation of eNOS in a Rat Model. Cell Physiol Biochem 2016;40:1163-1174.

7 Vilahur G, Badimon L: Ischemia/reperfusion activates myocardial innate immune response: The key role of the toll-like receptor. Front Physiol 2014;5:496.

8 Mann DL: The emerging role of innate immunity in the heart and vascular system. Circ Res 2011;108:11331145.

-9 Vilahur G, Juan-Babot O, Pena E, Onate B, Casani L, Badimon L: Molecular and cellular mechanisms involved in cardiac remodeling after acute myocardial infarction. J Mol Cell Cardiol 2011;50:522-533.

10 Kimoto M, Nagasawa K, Miyake K: Role of TLR4/MD-2 and RP105/MD-1 in innate recognition of lipopolysaccharide. Scand J Infect Dis 2003;35:568-572.

11 Omueti KO, Beyer JM, Johnson CM, Lyle EA, Tapping RI: Domain Exchange between Human Toll-like Receptors 1 and 6 Reveals a Region Required for Lipopeptide Discrimination. J Biol Chem 2005;280:36616 -36625.

12 Nagai Y, Kobayashi T, Motoi Y, Ishiguro K, Akashi S, Saitoh S, Kusumoto Y: The radioprotective 105/ MD-1 complex links TLR2 and TLR4/MD-2 in antibody response to microbial membranes. J Immunol 2005;174:7043-7049.

13 Liu B, Zhang N, Liu Z, Fu Y, Feng S, Wang S, Cao Y, Li D, Liang D, Li F, Song X, Yang Z: RP105 involved in activation of mouse macrophages via TLR2 and TLR4 signaling. Mol Cell Biochem 2013;378:183-193.

14 Schultz TE,BlumenthalA:The RP105/MD-1 complex: Molecular signaling mechanisms and pathophysiological implications. J Leukocyte Biol 2017;101:183-192.

15 Zuo Y, Wang Y, Hu H, Cui W. Atorvastatin protects myocardium against ischemia-reperfusion injury through inhibiting miR-199a-5p. Cell Physiol Biochem 2016;39(3):1021-1030.

16 Hullinger TG, Montgomery RL, Seto AG, Dickinson BA, Semus HM, Lynch JM, Dalby CM, Robinson K, Stack C, Latimer PA, Hare JM, Olson EN, van Rooij E: Inhibition of miR-15 protects against cardiac ischemic InjuryNovelty and significance. Circ Res 2012;110:71-81.

17 Hinkel R, Penzkofer D, Zuhlke S, Fischer A, Husada W, Xu QF, Baloch E, van Rooij E, Zeiher AM, Kupatt C, Dimmeler S: Inhibition of microRNA-92a protects against ischemia/reperfusion injury in a large-animal model. Circulation 2013;128:1066-1075. 


\section{Cellular Physiology Cell Physiol Biochem 2018;49:1090-1104 \begin{tabular}{ll|l|l|} 
and Biochemistry 2018 The Author(s). Published by S. Karger AG, Basel \\
\cline { 2 - 3 }
\end{tabular}}

Yang et al.: Down-Regulation of Mir-327 Attenuates MIRI

18 Shi J, Bei Y, Kong X, Liu X, Lei Z, Xu T, Wang H, Xuan Q, Chen P, Xu J, Che L, Liu H, Zhong J, Sluijter JP, Li $\mathrm{X}$, Rosenzweig A, Xiao J: MiR-17-3p contributes to Exercise-Induced cardiac growth and protects against myocardial Ischemia-Reperfusion injury. Theranostics 2017;7:664-676.

19 Hu S, Huang M, Li Z, Jia F, Ghosh Z, Lijkwan MA, Fasanaro P, Sun N, Wang X, Martelli F, Robbins RC, Wu JC: MicroRNA-210 as a novel therapy for treatment of ischemic heart disease. Circulation 2010;122:S124-S131.

20 Zhu J, Yao K, Wang Q, Guo J, Shi H, Ma L, Liu H, Gao W, Zou Y, Ge J. Ischemic postconditioning-regulated miR499 protects the rat heart against ischemia/reperfusion injury by inhibiting apoptosis through PDCD4. Cell Physiol Biochem 2016;39:2364-2380.

-21 Wang XH, Qian RZ, Zhang W, Chen SF, Jin HM, Hu RM: MicroRNA-320 expression in myocardial microvascular endothelial cells and its relationship with insulin-like growth factor-1 in type 2 diabetic rats. Clin Exp Pharmacol P 2010;36:181-188.

22 Mukhopadhyay P, Das S, Ahsan MK, Otani H, Das DK: Modulation of microRNA 20b with resveratrol and longevinex is linked with their potent anti-angiogenic action in the ischaemic myocardium and synergestic effects of resveratrol and $\gamma$-tocotrienol. J Cell Mol Med 2012;16:2504-2517.

-23 Wang JF, Zha YF, Li HW, Wang F, Bian Q, Lai XL, Yu G: Screening plasma miRNAs as biomarkers for renal ischemia-reperfusion injury in rats. Med Sci Monit 2014;20:283-289.

24 Liang-Hui L I, Chen W H, Zheng H: An optimized modification of TTC staining method for better detection of myocardial ischemia-reperfusion injury in rats [J]. Acta Lab Anim Sci Sinica, 2014;22:75-78.

25 Ha T, Liu L, Kelley J, Kao R, Williams D, Li C: Toll-Like receptors: New players in myocardial Ischemia/ Reperfusion injury. Antioxid Redox Sign 2011;15:1875-1893.

-26 Oyama J, Jr B C, Liu X: Reduced myocardial Ischemia-Reperfusion injury in Toll-Like receptor 4-Deficient mice. Circulation 2004;109:784-789.

27 Hua F, Ha T, Ma J, Li Y, Kelley J, Gao X: Protection against Myocardial Ischemia/Reperfusion Injury in TLR4Deficient Mice Is Mediated through a Phosphoinositide 3-Kinase-Dependent Mechanism. J Immunol 2007;178:7317-7324.

28 Chong AJ, Shimamoto A, Hampton CR, Takayama H, Spring DJ, Rothnie CL, Yada M, Pohlman TH, Verrier ED: Toll-like receptor 4 mediates ischemia/reperfusion injury of the heart. J Thorac Cardiovasc Surg 2004;128:170-179.

29 Shimamoto A, Chong AJ, Yada M, Shomura S, Takayama H, Fleisig AJ, Agnew ML, Hampton CR, Rothnie CL, Spring DJ, Pohlman TH, Shimpo H, Verrier ED: Inhibition of Toll-like receptor 4 with eritoran attenuates myocardial ischemia-reperfusion injury. Circulation 2006;114:I270-I274.

-30 Liu L, Wang Y, Cao Z, Wang M, Liu X, Gao T, Hu Q, Yuan W, Lin L: Up-regulated TLR4 in cardiomyocytes exacerbates heart failure after long-term myocardial infarction. J Cell Mol Med 2015;19:2728-2740.

-31 Bagchi AK, Akolkar G, Mandal S, Ayyappan P, Yang X, Singal PK: Toll-like receptor 2 dominance over Toll-like receptor 4 in stressful conditions for its detrimental role in the heart. Am J Physiol-Heart C 2017;312:H1238-H1247.

-32 Liu Q, Li J, Jubair S, Wang D, Luo Y, Fan D, Janicki JS: Sparstolonin b attenuates Hypoxia-Induced apoptosis, necrosis and inflammation in cultured rat left ventricular tissue slices. Cardiovasc Drug Ther 2014;28:433439.

-33 Shishido T, Nozaki N, Yamaguchi S, Shibata Y, Nitobe J, Miyamoto T, Takahashi H, Arimoto T, Maeda K, Yamakawa M, Takeuchi O, Akira S, Takeishi Y, Kubota I: Toll-like receptor-2 modulates ventricular remodeling after myocardial infarction. Circulation 2003;108:2905-2910.

-34 Ha T, Hu Y, Liu L, Lu C, McMullen JR, Kelley J, Kao RL, Williams DL, Gao X, Li C: TLR2 ligands induce cardioprotection against ischaemia/reperfusion injury through a PI3K/Akt-dependent mechanism. Cardiovasc Res 2010;87:694-703.

-35 Nagai Y, Shimazu R, Ogata H: Requirement for MD-1 in cell surface expression of RP105/CD180 and B-cell responsiveness to lipopolysaccharide. Blood 2002;99:1699-1705.

-36 Yang J, Guo X, Yang J, Ding J, Li S, Yang R, Fan Z, Yang C: RP105 protects against apoptosis in Ischemia/ Reperfusion-Induced myocardial damage in rats by suppressing TLR4-Mediated signaling pathways. Cell Physio Biochem 2015;36:2137-2148.

-37 Louwe MC, Karper JC, de Vries MR, Nossent AY, Bastiaansen AJ, van der Hoorn JW, Willems VDK, Rensen PC, Steendijk P, Smit JW, Quax PH: RP105 deficiency aggravates cardiac dysfunction after myocardial infarction in mice. Int J Cardiol 2014;176:788-793. 
-38 Zhao Y, Wang Y, Gong J, Yang L, Niu C, Ni X, Wang Y, Peng S, Gu X, Sun C, Yang Y: Chitosan degradation products facilitate peripheral nerve regeneration by improving macrophage-constructed microenvironments. Biomaterials 2017;134:64-77.

-39 Yáñez-Mó M, Siljander PR, Andreu Z, Zavec AB, Borràs FE, Buzas EI, Buzas K, Casal E, Cappello F, Carvalho $\mathrm{J}$ : Biological properties of extracellular vesicles and their physiological functions. J Extracell Vesicles 2015;4:1-60.

40 de Couto G, Gallet R, Cambier L, Jaghatspanyan E, Makkar N, Dawkins JF, Berman BP, Marbán E: Exosomal microRNA Transfer into Macrophages Mediates Cellular Postconditioning. Circulation 2017;136:200-214.

-41 Li J, Rohailla S, Gelber N, Rutka J, Sabah N, Gladstone RA, Wei C, Hu P, Kharbanda RK, Redington AN: MicroRNA-144 is a circulating effector of remote ischemic preconditioning. Basic Res Cardiol 2014;109:115. 